\title{
Glucose regulated protein 78 promotes cell invasion via regulation of UPA production and secretion in colon cancer cells
}

\author{
Zongwei $\mathrm{Li}^{1, *}$, Lichao Zhang ${ }^{1, *}$, Hanqing $\mathrm{Li}^{2}$, Shuhua Shan ${ }^{1}$ \& Zhuoyu $\mathrm{Li}^{1,3, *}$ \\ ${ }^{1}$ Institute of Biotechnology, Key Laboratory of Chemical Biology and Molecular Engineering of National Ministry of Education, ${ }^{2}$ College of Life \\ Science, Shanxi University, Taiyuan, 030006, China, ${ }^{3}$ College of Life Science, Zhejiang Chinese Medical University, Hangzhou 310053, China
}

Glucose regulated protein 78 (GRP78) is frequently highly expressed in tumor cells, contributing to the acquisition of several phenotypic cancer hallmarks. GRP78 expression is also positively correlated with tumor metastasis, and promotes hepatocellular carcinoma cell invasion via increasing cell motility, however, other mechanisms involving the prometastatic roles of GRP78 remain to be elucidated. Here we report that forced GRP78 expression promotes colon cancer cell migration and invasion through upregulating MMP-2, MMP-9 and especially uPA production. These effects of GRP78 are mediated by enhancing the activation of $\beta$-catenin signaling. Interestingly, we identify that GRP78 interacts with uPA both in the cells and in the culture medium, suggesting that GRP78 protein is likely to directly facilitate uPA secretion via protein-protein interaction. Taken together, our findings demonstrate for the first time that besides stimulation of cell motility, GRP78 can act by increasing proteases production to promote tumor cell invasion. [BMB Reports 2014; 47(8): 445-450]

\section{INTRODUCTION}

Glucose regulated protein 78 (GRP78) is a molecular chaperone localized in the endoplasmic reticulum (ER), involved in correct protein folding and assembly, proteasomal degradation of misfolded protein, and ER $\mathrm{Ca}^{2+}$ binding (1). In adult animals, deregulation of GRP78 has a causal relationship with neurological disorders as well as tumor progression (2). The expression status of GRP78 is positively correlated with the metastasis of many types of tumors (3-5). Knockdown of GRP78 inhibits tumor cell invasion in vitro and metastasis in xenograft models $(6,7)$. However, the molecular mechanism

*Corresponding author. Tel: +86-351-7018268; Fax: +86-3517018268; E-mail: lzy@sxu.edu.cn

${ }^{\#}$ The first two authors contributed equally to this work.

http://dx.doi.org/10.5483/BMBRep.2014.47.8.211

Received 27 September 2013, Revised 19 October 2013, Accepted 22 November 2013

Keywords: GRP78, uPA, Invasion, $\beta$-catenin, Colon cancer underlying the metastasis-promoting effects of GRP78 remains to be fully elucidated.

We previously demonstrated that the cell surface GRP78 co-localizes with the urokinase receptor (UPAR) and $\beta 1$-integrin, which facilitates the transformation of plasminogen to plasmin by uPA and promotes cell migration and invasion (8). Given that GRP78 has been reported to be overexpressed in colon cancer (9), we turned to focus on investigating the roles of intracellular GRP78 in colon cancer migration and invasion. Furthermore, our studies were extended to identify the signaling mechanisms that mediated such actions of GRP78.

\section{RESULTS}

GRP78 expression promotes DLD1 cell migration and invasion To investigate the role of GRP78 expression in colon cancer metastasis, GRP78 was overexpressed in DLD1 cells by lentivirus gene transfer. The expression of GFP and GFP-GRP78 in infected DLD1 cells was determined by Western blotting analysis using a GFP-specific antibody (Fig. 1C). Overexpression of GRP78 in DLD1 cells significantly promoted cell migration and increased wound closure rate (Fig. $1 \mathrm{~A}$ and $1 \mathrm{~B}$ ). Furthermore, GRP78 overexpression also facilitated DLD1 cell invasion as demonstrated by the transwell invasion assays (Fig. 1D and 1E).

\section{GRP78 stimulates the expression of MMP-2, MMP-9 and uPA in DLD1 cells}

Matrix metalloproteinase 2 (MMP-2) and MMP-9 as well as urokinase-type plasminogen activator (UPA) have been implicated to play important roles in colon cancer cell invasion and metastasis $(10,11)$. The mRNA levels of MMP-2, MMP-9 and UPA in GFP-GRP78 expressing cells increased approximately 4-fold, 4-fold and 15-fold compared to those in GFP expressing cells (Fig. 2A). Consistently, knockdown of endogenous GRP78 expression in DLD1 cells by GRP78-specific shRNA (GRP78-shRNA) significantly reduced their mRNA levels (Fig. 2B).

Given that the uPA mRNA is more abundant than MMP-2 and MMP-9 mRNA (supplemental data), and that uPA usually acts upstream of MMP-2 and MMP-9 activation (12). The expression and secretion of uPA were determined by Western

ISSN: 1976-670X (electronic edition)

Copyright (C) 2014 by the The Korean Society for Biochemistry and Molecular Biology

c) This is an open-access article distributed under the terms of the Creative Commons Attribution Non-Commercial License (http://creativecommons.org/licenses/by-nc/3.0) which permits unrestricted non-commercial use, distribution, and reproduction in any medium, provided the original work is properly cited. 


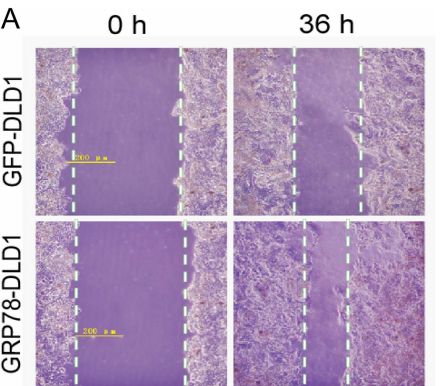

D GFP-DLD1 GRP78-DLD1

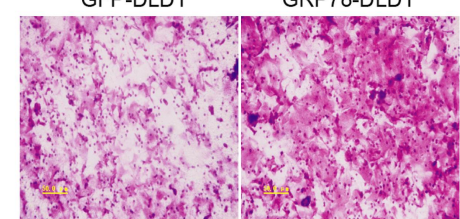

B
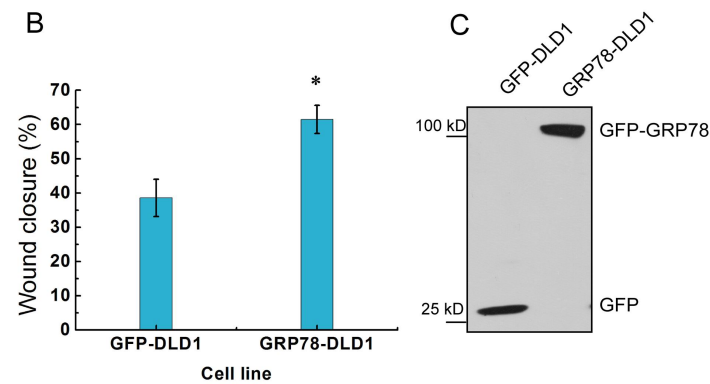

$\mathrm{E}$

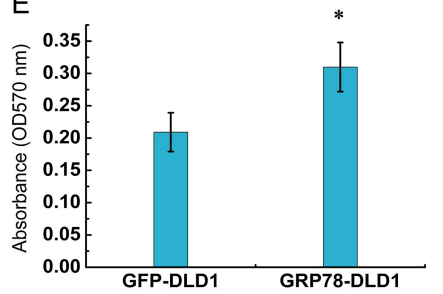

Fig. 1. GRP78 expression promotes DLD1 cell migration and invasion. (A) The cell migration dynamics of DLD1 cells stably expressing GFP (GFPDLD1) and GFP-GRP78 (GRP78-DLD1) by wound healing assay. (B) The statistical graph of wound closure rate of result A. ${ }^{*} \mathrm{P}<0.05$ versus GFPDLD1. (C) Western blotting detection of GFP in GFP-DLD1 and GRP78DLD1 cells. (D) Cell invasion of GFPDLD1 and GRP78-DLD1 cells was assayed by the matrigel-transwell assay. (E) The statistical graph of cell invasion changes of result D. $* \mathrm{P}<$ 0.05 versus GFP-DLD1.
A
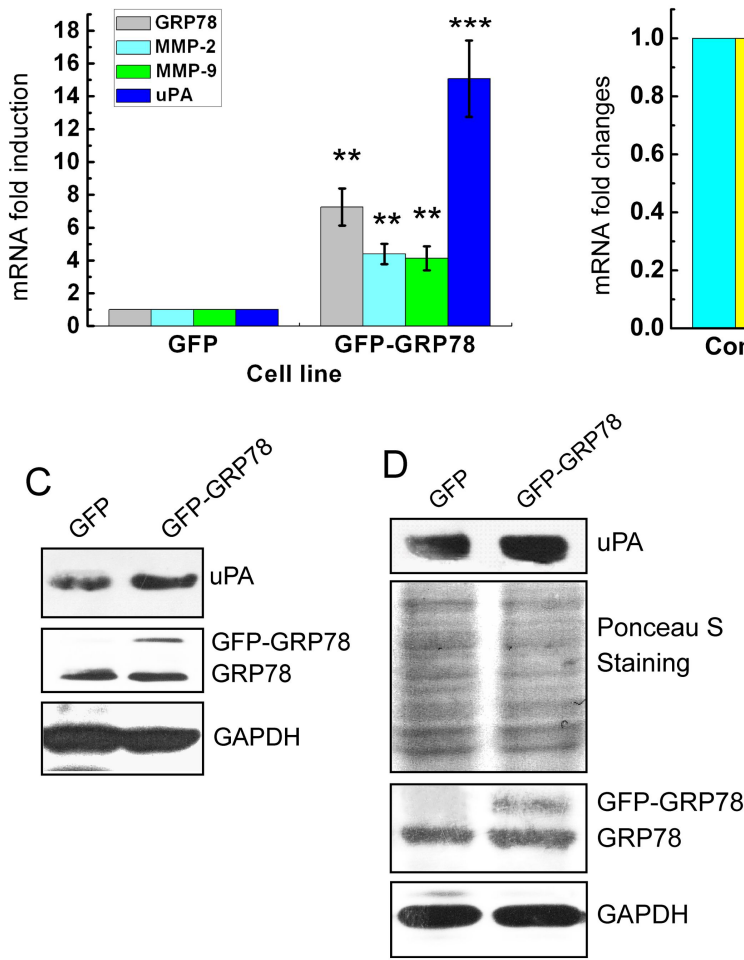

blotting of the whole cell lysates and culture media, respectively. As shown in Fig. 2C and 2D, GRP78 overexpression increased both the protein expression and the extracellular release of uPA. In addition, gelatin zymography assay showed that overexpression of GRP78 increased MMP-2 activity in the medium (Fig. 2E). These results demonstrate that the proinva-
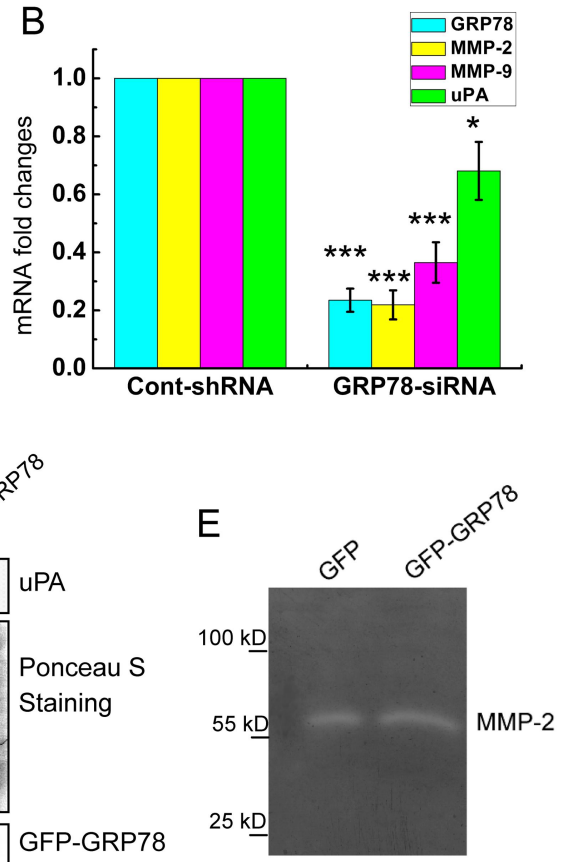

Fig. 2. GRP78 stimulates the expression of MMP-2, MMP-9 and UPA in DLD1 cells. (A) Relative mRNA levels of GRP78, MMP-2, MMP-9 and uPA in GFP-DLD1 and GRP78-DLD1 cells. ${ }^{* * P}<0.01,{ }^{* * * P}<0.001$ vs GFP-DLD1. (B) Relative mRNA levels of MMP-2, MMP-9, UPA and GRP78 in GRP78 knockdown DLD1 cells. *P $<$ $0.05, * * * P<0.001$ vs Cont-shRNA (C) Western blots of UPA and GRP78 in GFP-DLD1 and GRP78-DLD1 cells. (D) Western botting analysis of uPA secretion in the conditioned medium from GFP-DLD1 and GRP78-DLD1 cells. (E) Zymographic analysis of MMP-2 and MMP-9 activities in conditioned medium from GFP-DLD1 and GRP78-DLD1 cells. sion effect of GRP78 is most likely mediated by upregulation of MMP-2, MMP-9 and uPA production.

GRP78 promotes uPA expression via the $\beta$-catenin pathway We have found that GRP78 overexpression stimulated the activation of HIF-1 $\alpha$ and AKT signaling pathways (our unpublished 
A

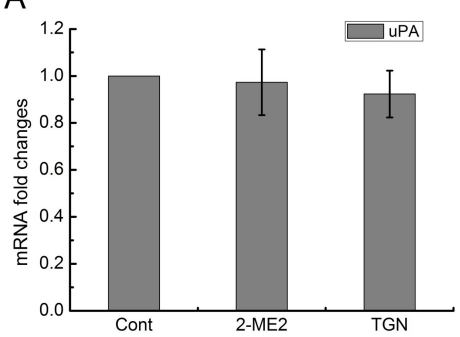

$\mathrm{D}$



$\mathrm{C}$

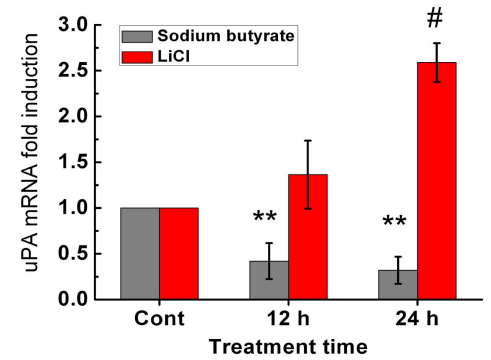

E

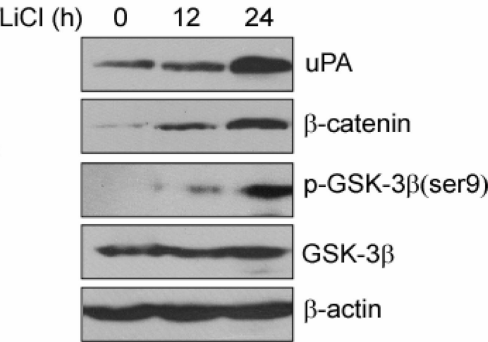

Fig. 3. GRP78 promotes uPA expression via the $\beta$-catenin pathway. (A) Relative mRNA levels of uPA in GRP78-DLD1 cells in the presence of the HIF-1 $\alpha$ inhibitor 2-ME2 (50 $\mu \mathrm{M})$ or the Akt inhibitor TCN $(10 \mu \mathrm{M})$. (B) Western blots of $\beta$-catenin in GFP-DLD1 and GRP78-DLD1 cells. (B) Relative mRNA levels of uPA in DLD1 cells treated by sodium butyrate and lithium chloride for the indicated time intervals. $* \mathrm{P}<0.05$, ${ }^{\#} \mathrm{P}$ $<0.05$ and $* * P<0.01$ versus Cont. (D) Western blots of UPA and $\beta$-catenin in DLD1 cells treated by sodium butyrate for the indicated time intervals. (E) Western blots of

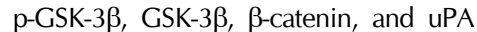
in DLD1 cells treated with $\mathrm{LiCl}(10$ $\mathrm{mM}$ ) for the indicated hours. data), both of which have been reported to be able to regulate uPA expression $(13,14)$. To identify the mechanism underlying the upregulation of UPA expression by GRP78, the HIF- $1 \alpha$ inhibitor 2-methoxyestradiol (2-ME2) and the Akt inhibitor triciribine (TCN) were applied to block the corresponding signaling pathways, and the results showed that neither 2-ME2 nor TCN could affect the expression of uPA (Fig. 3A). Our results also showed that GRP78 overexpression significantly raised the protein level of $\beta$-catenin (Fig. 3B), which has been reported to contribute to the transactivation of UPA (11). Thus lithium chloride ( $\mathrm{LiCl}$ ) and sodium butyrate (SB) were used to respectively activate and suppress the $\beta$-catenin signaling. $\mathrm{LiCl}$ treatment resulted in activation of the $\mathrm{Wnt} / \beta$-catenin signaling via mocking GSK-3 $\beta$ inhibition, accompanied by increased uPA mRNA and protein expression (Fig. $3 \mathrm{C}$ and $3 \mathrm{E}$ ). Convertsely, treatment with sodium butyrate caused the inhibition of $\beta$-catenin signaling and the consequent decreased uPA expression (Fig. 3C and 3D). The results above suggest that induction of uPA expression by GRP78 depends on the activation of $\beta$-catenin signaling pathway.

\section{GRP78 interacts with uPA and facilities its secretions in colon cancer cells}

GRP78 has been identified as a cell surface receptor for the Kringle 5 domain of plasminogen (15). UPA and plasminogen shared a similar protein domain structure, as determined using the program available at Scansite (http://www.scansite.mit.edu) (Fig. 4A). Thus we speculated that GRP78 may interact with UPA in colon cancer cells. To test this possibility, immunocytochemical staining of GRP78 and UPA in DLD1 and HT-29 cells was carried out. As shown in Fig. 4B, GRP78 colocalized with UPA in both cells. Consistent with the immunocytochemical results, co-immunoprecipitiation (Co-IP) assays showed that GRP78 complexed with uPA in both HT-29 and DLD1 cells (Fig. 4C). GST-pulldown assays further demonstrated that purified recombinant GST-GRP78 protein bound to the UPA protein from the extracts of DLD1 cells (Fig. 4D). Moreover, GRP78 also co-immunoprecipitated with uPA in the medium from DLD1 cells (Fig. 4E). These data suggest that GRP78 may directly facilitate the extracelluar secretion of uPA by means of protein-protein interaction.

\section{DISCUSSION}

Two characteristics of highly malignant cells are their increased motility and secretion of proteases, allowing these cells to penetrate surrounding basement membranes and metastasize. Overexpression of GRP78 enhances the activation of focal adhesion kinase (FAK), accelerates the process of cell spreading, and promotes cell motility in hepatocellular carcinoma (16). In this study, we note that GRP78 overexpression also promotes cell migration and invasion in colon cancer cells, and these actions of GRP78 are most likely attributed to the increased production of MMP-2, MMP-9 and uPA. Thus GRP78 can act through both stimulation of cell motility and increase of proteases production to promote tumor cell migration and invasion.

The uPA-plasmin system has been reported to be implicated in zymogen proteases activation, including MMP-2 and MMP-9 (12). The mRNA abundance of uPA is substantially 
A

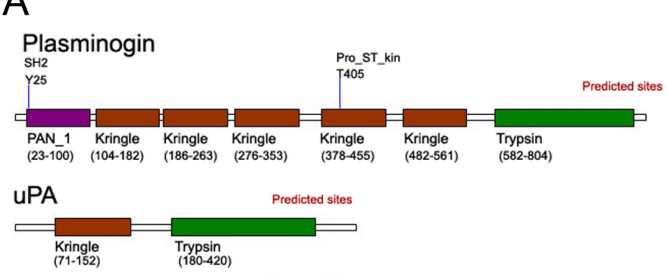

B

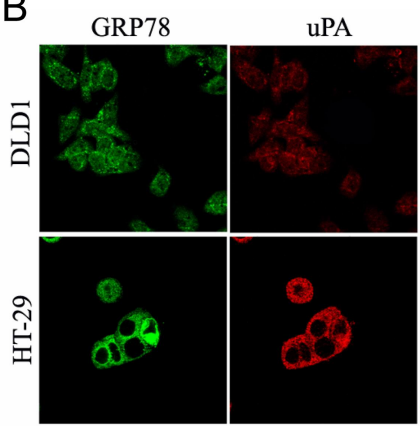

D

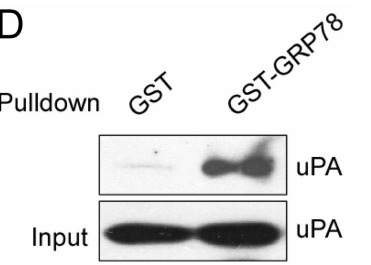

C

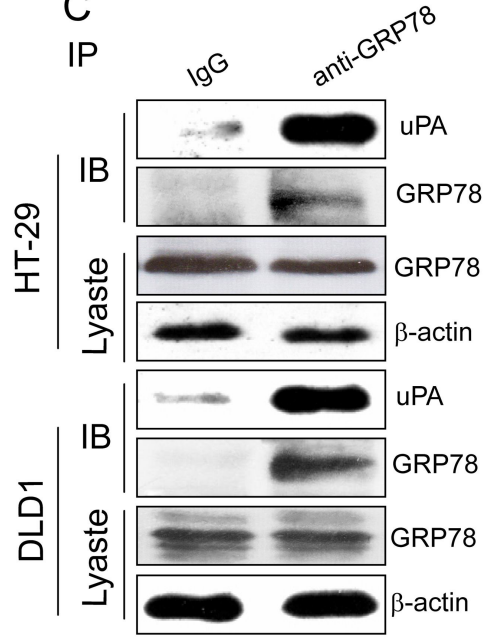

Fig. 4. GRP78 interacts with $\mathrm{UPA}$ in colon cancer cells and their secretions. (A) Protein domain prediction of uPA and plasminogen using the Scansite program (http://www.scansite.mit.edu) (B) HT-29 and DLD1 cells were stained with antibodies against GRP78 and UPA, and visualized with FITCand TRITC-conjugated secondary antibodies, respectively. The yellow dots correspond to spots where GRP78 (green) and UPA (red) colocalized. (C) Analysis of GRP78 and UPA interactions. Immunoprecipitation experiments were carried out on HT-29 (top) and DLD1 (bottom) lysates using GRP78 antibodies followed by Western blotting for UPA. (D) GST-GRP78 pulldown of lysates from DLD1 cells. The precipitates were separated by SDSPAGE and immunoblotted with anti-uPA antibody. (E) Analysis of GRP78 and uPA interactions in conditioned medium. Immunoprecipitation experiments were performed on serum free conditioned medium from DLD1 cells using GRP78 antibody followed by Western blotting for uPA.

higher than that of MMP-2 and MMP-9 in colon cancer cells, suggesting that UPA may serve as the major proteases for the initiation of cell migration and invasion. Aberrant activation of $\beta$-catenin-TCF signaling, resulting from mutations in $\beta$-catenin or its degradation machinery, is a characteristic to early stages of colorectal carcinoma development (17). High expression of GRP78 in colon cancer cells is likely to further enhance the $\beta$-catenin signaling, concurrently accompanied by higher uPA production. In this sense, it is reasonable to deduce that colon cancer cells with higher GRP78 expression are endowed with higher $\beta$-catenin signaling activity and more uPA production, and thus with a higher metastatic ability. This may be one of the reasons why there is higher GRP78 expression in metastatic tissues.

GRP78 can be secreted into the extracelluar microenvironment by solid tumors, acting as a signaling molecule within the tumor milieu $(18,19)$. Interestingly, we identify that GRP78 interacts with uPA both in the cells and in the culture medium. These results suggest that GRP78 protein is likely to directly facilitate UPA secretion via protein-protein interaction. Moreover, since GRP78 can bind the Kringle 5 domain of plasminogen (15), the enzymatic substrate of UPA, interaction of GRP78 with UPA in tumor microenvironment will lead to the close location of UPA and plasminogen, more efficient transformation of plasminogen to plas- min, and final acceleration of tumor cell invasion.

\section{MATERIALS AND METHODS}

\section{Materials}

The medium and fetal bovine serum (FBS) were purchased from Invitrogen (Carlsbad, CA). Trizol, PrimeScript RT Master Mix and SYBR green PCR master mix were from Takara (Shiga, Japan). GRP78 and uPA antibodies were from Abcam (Cambridge, UK). $\beta$-actin antibody was from Bioworld Technology (Minneapolis, MN). GAPDH and GFP antibodies were from Sangon Biotech (Shanghai, China). $\beta$-catenin antibody was obtained from Abmart (Shanghai, China). FITC-, TRITC- and HRP-conjugated secondary antibodies were obtained from Invitrogen (Carlsbad, CA).

\section{Cell culture and lentivirus infection}

Human colon carcinoma HT29 and DLD1 cell lines were obtained from the American Type Culture Collection and cultured in RPMI-1640 medium containing 10\% FBS. For stable cell line selection, human GRP78 cDNA was subcloned into the pLVX-AcGFP1-N1 Vector (Clontech). GRP78 shRNAs constructed in pLKO.1-Puro were purchased from Sigma (Mission shRNA). GRP78 overexpression/shRNA plasmid, psPAX2 and 
pMD2.G were co-transfected into 293T cells at 15:10:5 g. Media containing virus was collected and concentrated using $100 \mathrm{kDa}$ ultrafiltration membranes (Millipore). DLD1 cells were infected with the viruses in the presence of polybrene (8 $\mu \mathrm{g} / \mathrm{ml}$ ) for $24 \mathrm{~h}$, and then subjected to selection by $5 \mu \mathrm{g} / \mathrm{ml}$ puromycin. Hairpin sequences in these shRNA constructs are depicted as follows: Table 1 shRNA sequences used in this study: GRP78-1: 5'-CCGGAGATTCAGCAACTGGTTAAAGCT CGAGCTTTAACCAGTTGCTGAATCTTTTTTG-3'; GRP78-2: 5'CCGGGAGCGCATTGATACTAGAAATCTCGAGATTTCTAGTA TCAATGCGCTCTTITTG-3'; Control: 5'-CCGGCCTAAGGTTA AGTCGCCCTCGCTCGAGCGAGGGCGACTTAACCTTAGGTT TTTG-3'.

\section{RNA extraction and real-time PCR analysis}

Total RNA extraction and real-time PCR analysis were performed as previously described (8). The primers used in this study were depicted in as follows: MMP-2-F: CTCATCGCAGA TGCCTGGAA, MMP-2-R: CAGCCTAGCCAGTCGGATTTG; MMP-9-F: ACGCACGACGTCTTCCAGTA, MMP-9-F: CCACCT GGTTCAACTCACTCC; UPA-F: GCTGCTGACCCACAGTGGA A,uPA-R: AAAGTCATGCGGCCTTGGAG; GRP78-F: CTGTGC AGCAGGACATCAAGTTC, GRP89-R: TGTTTGCCCACCTCCA ATATCA; GAPDH-F: GCACCGTCAAGGCTGAGAAC; GAPDHR: TGGTGAAGACGCCAGTGGA.

\section{Westem blotting, co-immunoprecipitation and GST-pulldown assays \\ Western blotting and co-immunoprecipitation assays were per- formed as previously described (8). For the GST-pulldown as- says, GST-GRP78 or GST immobilized on Sepharose 4B-gluta- thione beads (GE Healthcare) were incubated with the whole cell lysates overnight at $4^{\circ} \mathrm{C}$ followed by washing with the lysis buffer. The bound proteins were dissolved in $2 \times$ SDS loading buffer, separated by $10 \%$ SDS-PAGE, and immunoblotted with the uPA antibody.}

\section{Immunofluorescence analysis}

Cells were plated on 6-well glass slides. After appropriate treatments, the cells were fixed in $4 \%$ paraformaldehyde in PBS for 30 min, and permeabilized with $0.3 \%$ Triton X-100 in PBS for 10 min. Next, the slides were blocked in $2 \%$ goat serum for 1 $\mathrm{h}$ and incubated with the primary antibodies at $4^{\circ} \mathrm{C}$ overnight. The slides were then washed and incubated with the appropriate secondary antibodies. After three PBS washes, the slides were mounted in gelvatol for confocal immunofluorescence analysis.

\section{Cell migration, invasion and gelatin zymography assays}

For cell migration assay, DLD1 cells were seed into a 24-well plate and the confluent cell monolayer was scratched with a plastic pipette tip to create a cell free zone in the well. Photographs were taken immediately after wound incision and at later time points. For cell invasion assay, cells were placed a
Matrigel-coated transwell chamber (BD Biosciences) at $2.0 \times$ $10^{5}$ per insert in serum-free medium and were allowed to migrate toward $15 \%$ FBS for $48 \mathrm{~h}$. After that, non-invading cells on the top of the membrane were removed by scraping. Invaded cells on the bottom of the membrane were fixed and stained with crystal violet. The stained membranes were solubilized by the addition of $1 \%$ SDS, and the number of invaded cells was determined using a microplate reader at $570 \mathrm{~nm}$. Activities of MMP-2 and MMP-9 in conditioned medium from GFP-DLD1 and GRP78-DLD1 cells were examined by gelatin zymography according to the method reported in the literature (20).

\section{Statistical analysis}

Data are expressed as the mean \pm SEM. Differences among groups were tested by one-way analysis of variance (ANOVA). Comparisons between two groups were evaluated using Student's t-test. A value of $\mathrm{P}<0.05$ was considered statistically significant.

\section{ACKNOWLEDGEMENTS}

This study was supported by the National Natural Science Foundation of China (No. 31271516, No. 31201072), Shanxi Province Science Foundation for Youths (2012021028-4), Research Fund for the Doctoral Program of Higher Education of China (20111401110011).

\section{REFERENCES}

1. Li, X., Zhang, K. and Li, Z. (2011) Unfolded protein response in cancer: the physician's perspective. J. Hematol. Oncol. 4, 8.

2. Wang, M., Wey, S., Zhang, Y., Ye, R. and Lee, A. S. (2009) Role of the unfolded protein response regulator GRP78/BiP in development, cancer, and neurological disorders. Antioxid. Redox Signal. 11, 2307-2316.

3. Zhang, J., Jiang, Y., Jia, Z., Li, Q., Gong, W., Wang, L., Wei, D., Yao, J., Fang, S. and Xie, K. (2006) Association of elevated GRP78 expression with increased lymph node metastasis and poor prognosis in patients with gastric cancer. Clin. Exp. Metastasis 23, 401-410.

4. Zheng, H. C., Takahashi, H., Li, X. H., Hara, T., Masuda, S., Guan, Y. F. and Takano, Y. (2008) Overexpression of GRP78 and GRP94 are markers for aggressive behavior and poor prognosis in gastric carcinomas. Hum. Pathol. 39, 1042-1049.

5. Sun, Q., Hua, J., Wang, Q., Xu, W., Zhang, J., Kang, J. and Li, M. (2012) Expressions of GRP78 and Bax associate with differentiation, metastasis, and apoptosis in non-small cell lung cancer. Mol. Biol. Rep. 39, 6753-6761.

6. Fu, Y. and Lee, A. S. (2006) Glucose regulated proteins in cancer progression, drug resistance and immunotherapy. Cancer Biol. Ther. 5, 741-744.

7. Chiu, C. C., Lin, C. Y., Lee, L. Y., Chen, Y. J., Kuo, T. F., Chang, J. T., Liao, C. T., Wang, H. M., Yen, T. C., Shen, C. R., Liao, S. K. and Cheng, A. J. (2008) Glucose-regulated 
protein 78 regulates multiple malignant phenotypes in head and neck cancer and may serve as a molecular target of therapeutic intervention. Mol. Cancer Ther. 7, 2788-2797.

8. Li, Z., Zhang, L., Zhao, Y., Li, H., Xiao, H., Fu, R., Zhao, C. and Wu, H. (2013) Cell-surface GRP78 facilitates colorectal cancer cell migration and invasion. Int. J. Biochem. Cell Biol. 45, 987-994.

9. Xing, X., Lai, M., Wang, Y., Xu, E. and Huang, Q. (2006) Overexpression of glucose-regulated protein 78 in colon cancer. Clin. Chim. Acta. 364, 308-315.

10. Mook, O. R., Frederiks, W. M. and Van Noorden, C. J. (2004) The role of gelatinases in colorectal cancer progression and metastasis. Biochim. Biophys. Acta. 1705, 69-89.

11. Hiendlmeyer, E., Regus, S., Wassermann, S., Hlubek, F., Haynl, A., Dimmler, A., Koch, C., Knoll, C., van Beest, M., Reuning, U., Brabletz, T., Kirchner, T. and Jung, A. (2004) Beta-catenin up-regulates the expression of the urokinase plasminogen activator in human colorectal tumors. Cancer Res. 64, 1209-1214.

12. Blasi, F. and Carmeliet, P. (2002) uPAR: a versatile signalling orchestrator. Nat. Rev. Mol. Cell Biol. 3, 932-943.

13. Tacchini, L., Dansi, P., Matteucci, E. and Desiderio, M. A. (2001) Hepatocyte growth factor signalling stimulates hypoxia inducible factor-1 (HIF-1) activity in HepG2 hepatoma cells. Carcinogenesis 22, 1363-1371.

14. Agarwal, A., Das, K., Lerner, N., Sathe, S., Cicek, M., Casey, G. and Sizemore, N. (2005) The AKT/I kappa B kinase pathway promotes angiogenic/metastatic gene expression in colorectal cancer by activating nuclear factor-kappa B and beta-catenin. Oncogene 24, 1021-1031.

15. Davidson, D. J., Haskell, C., Majest, S., Kherzai, A., Egan, D. A., Walter, K. A., Schneider, A., Gubbins, E. F., Solomon, L., Chen, Z., Lesniewski, R. and Henkin, J. (2005) Kringle 5 of human plasminogen induces apoptosis of endothelial and tumor cells through surface-expressed glucose-regulated protein 78. Cancer Res. 65, 4663-4672.

16. Su, R., Li, Z., Li, H., Song, H., Bao, C., Wei, J. and Cheng, L. (2010) Grp78 promotes the invasion of hepatocellular carcinoma. BMC Cancer 10, 20.

17. Morin, P. J., Sparks, A. B., Korinek, V., Barker, N., Clevers, H., Vogelstein, B. and Kinzler, K. W. (1997) Activation of beta-catenin-Tcf signaling in colon cancer by mutations in beta-catenin or APC. Science 275, 1787-1790.

18. Li, Z. (2012) Glucose regulated protein 78: a critical link between tumor microenvironment and cancer hallmarks. Biochim. Biophys. Acta. 1826, 13-22.

19. Kern, J., Untergasser, G., Zenzmaier, C., Sarg, B., Gastl, G., Gunsilius, E. and Steurer, M. (2009) GRP-78 secreted by tumor cells blocks the antiangiogenic activity of bortezomib. Blood 114, 3960-3967.

20. Yoshioka, I., Tsuchiya, Y., Aozuka, Y., Onishi, Y., Sakurai, H., Koizumi, K., Tsukada, K. and Saiki, I. (2005) Urinary trypsin inhibitor suppresses surgical stress-facilitated lung metastasis of murine colon 26-L5 carcinoma cells. Anticancer Res. 25, 815-820. 